OPEN ACCESS

Edited by:

Muthupandian Saravanan,

Saveetha Dental College

and Hospitals, India

Reviewed by:

Balakumar Chandrasekaran, Philade/phia University, Jordan Hamed Barabadi,

Shahid Beheshti University of Medical Sciences, Iran

Yugal Kishore Mohanta, North Orissa University, India

*Correspondence: Yaling $\mathrm{Li}$ lylapothecary@swmu.edu.cn

Specialty section: This article was submitted to Pharmacology of Anti-Cancer Drugs, a section of the journal Frontiers in Oncology

Received: 15 April 2021 Accepted: 08 June 2021 Published: 25 June 2021

Citation:

Wang J, Su S, Li J and Li Y (2021) Efficacy and Safety of Camrelizumab

Monotherapy and Combination Therapy for Cancers: A Systematic Review and Meta-Analysis. Front. Oncol. 11:695512. doi: 10.3389/fonc.2021.695512

\section{Efficacy and Safety of Camrelizumab Monotherapy and Combination Therapy for Cancers: A Systematic Review and Meta-Analysis}

\author{
Jiting Wang ${ }^{1,2}$, Song $\mathrm{Su}^{3}$, Jun $\mathrm{Li}^{4}$ and Yaling $\mathrm{Li}^{2 *}$ \\ 1 Department of Pharmacy, Southwest Medical University, Luzhou, China, ${ }^{2}$ Department of Pharmacy, Affiliated Hospital of \\ Southwest Medical University, Luzhou, China, ${ }^{3}$ Department of Hepatobiliary Surgery, Affiliated Hospital of Southwest Medical \\ University, Luzhou, China, ${ }^{4}$ Department of Anorectal, Affiliated Hospital of Southwest Medical University, Luzhou, China
}

Objective: This meta-analysis compared the safety and efficacy of camrelizumab monotherapy and combination therapy, aiming to provide a reference for the clinical combined use of camrelizumab in the treatment of cancers and also provide a reference for the development of subsequent indications of camrelizumab.

Methods: Meta-analysis was used to analyze the four eligible literatures. Primary endpoints of effectiveness index were objective response rate (ORR), progression-free survival (PFS), control rate (CR). Primary endpoint of safety index was rating of severity of adverse drug reactions (grades 1-5).

Results: The ORR, PFS, and CR values of combined treatment with camrelizumab was better than alone treatment, camrelizumab alone was better than chemotherapy $(R R=0.45$; 95\% Cl, 0.30-0.67; $\mathrm{P}<0.001 ; \mathrm{RR}=1.63 ; 95 \% \mathrm{Cl}, 1.25-2.13 ; \mathrm{P}<0.001 ; \mathrm{RR}=0.73 ; 95 \% \mathrm{Cl}$, $0.52-1.02 ; \mathrm{P}<0.001)$. When grade $>2$, the incidence rate of combined treatment and chemotherapy are higher than monotherapy $(R R=0.66 ; 95 \% \mathrm{Cl}, 0.51-0.86 ; \mathrm{P}<0.001)$. In any grade, the safety of camrelizumab combination therapy was better than that of monotherapy, the safety of chemotherapy was better than camrelizumab plus chemotherapy.

Conclusion: In terms of effectiveness, the combination of camrelizumab is better than monotherapy, and monotherapy is better than chemotherapy. In terms of safety, when the grade $>2$, single use is better than combination therapy and chemotherapy. In any grade of adverse event, the safety of combined use of camrelizumab is better than that of single use, and the safety of chemotherapy is better than the combined use of camrelizumab plus chemotherapy.

Keywords: camrelizumab, efficacy, safety, monotherapy, combination therapy, meta-analysis

\section{INTRODUCTION}

Malignant tumors have become a serious threat to human life and health, and it is on the rise in recent years. The treatment of tumors have developed from surgery, radiotherapy, chemotherapy, local treatment, and now to molecular targeted therapy, and quickly entered the era of immunotherapy, effectively extending the overall survival of patients (1-3). Tumor immunotherapy use immune 
blockade inhibitors, also known as immune checkpoint inhibitors (anti-programmed cell death 1 [PD-1] or anti-programmed cell death ligand 1 [PD-L1] inhibitor, ICI), are a new type of monoclonal antibody drug that can inhibit the function of inhibitory immune receptors in order to evoke an immune antineoplastic response (4). In recent years, with the success of pembrolizumab and nivolumab, which target PD-1, and atezolizumab, durvalumab, and avelumab, which target PD-L1, ICI have been extensively studied in various tumor types (5).

Camrelizumab (SHR-1210) is a humanized monoclonal antibody against PD-1 independently developed by China. It showed high affinity for PD-1 $(\mathrm{KD}=3.31 \mathrm{nmol} / \mathrm{L})$ and high receptor occupancy on circulating $\mathrm{T}$ lymphocytes (85\% at a dose of $200 \mathrm{mg}$ ) (6). Phase 1 clinical trials showed that camrelizumab was well tolerated in patients with advanced solid tumors and showed antitumor activity (7-9). In China, camrelizumab has been approved for several indications including Hodgkin's lymphoma (NHL), advanced hepatocellular carcinoma (HCC), non-small cell lung cancer (NSCLC), and esophageal cancer (EC) (10). In addition, many clinical trials have confirmed that camrelizumab has a significant effect on many other cancers, including nasopharyngeal carcinoma (NPC), gastric cancer, pancreatic cancer, renal cancer, cervical cancer, and so on (11, 12). Although it is known that camrelizumab has a clinical therapeutic effect on a variety of tumors and also has a good development prospect, whether monotherapy or combination therapy is more effective and safe still needs to be explored. In this study, combination therapy means camrelizumab plus chemotherapy. Chemotherapy drugs include gemcitabine, cisplatin, decitabine, docetaxel, irinotecan, carboplatin, and pemetrexed, which are common and mature chemotherapeutic drugs that are used in strict accordance with the guidelines and instructions. It is of practical significance to study the safety and effectiveness of camrelizumab combined with these chemotherapy drugs and to compare with camrelizumab alone treatment.

Recently, the office of Orphan Products Development (OOPD) of the US Food and Drug Administration (FDA) awarded orphan drug design (ODD) for camrelizumab injection (13). The orphan drug qualification is based on an international multicenter phase III clinical study of first-line treatment of advanced HCC with camrelizumab combined with apatinib in the US, which has been carried out in 13 countries and regions including the US, Europe, South Korea, and China (14). After obtaining the orphan drug qualification, the clinical trials and market registration can be accelerated. Moreover, PubMed and other literature search websites did not find a meta-analysis of the comparison between single drug and combination drug of camrelizumab, so this study has certain importance and innovation. In this study, a total of five types of cancer, HCC, NPC, EC, NHL, and NSCLC, were included. The reason why HCC is not included is that the randomizedcontrolled trial of camrelizumab combined with apatinib in the treatment of HCC has only published a study protocol, but has not yet produced results (15). This meta-analysis compared the safety and efficacy of camrelizumab monotherapy and combination therapy, aiming to provide a reference for the clinical combined use of camrelizumab in the treatment of cancers and also provide a reference for the development of subsequent indications of camrelizumab.

\section{MATERIALS AND METHODS}

\section{Data Sources and Searches}

Embase, MEDLINE/Ovid, Epistemonikos, and Cochrane were searched from inception until January 13, 2021. In addition, we also manually searched for articles that met the criteria.

The question for this review was developed using the PICO criteria:

- Population: people aged 18 to 75 years, with NPC, EC, NHL, and NSCLC.

- Intervention: camrelizumab monotherapy or camrelizumab combined chemotherapy.

- Comparator: Camrelizumab + gemcitabine + cisplatin vs camrelizumab; camrelizumab + decitabine vs camrelizumab; camrelizumab vs docetaxel or irinotecan; camrelizumab + carboplatin + pemetrexed $v s$ carboplatin + pemetrexed.

- Outcome: ORR, PFS, CR, adverse event rate (grades 1-5).

\section{Study Selection}

We included peer-reviewed systematic reviews with metaanalyses of prospective longitudinal design (i.e., prospective/ cohort or retrospective/case-control) studies, as well as randomized-controlled trials (RCTs) that evaluated the efficacy and safety of camrelizumab monotherapy or combined therapy. The criteria are as follows:

Inclusion criteria:

- Meta-analyses that included people aged 18 to 75 years with camrelizumab in a single-drug group or combination therapy group.

- Meta-analyses of prospective longitudinal design studies that explored the efficacy and safety of camrelizumab monotherapy and combined therapy.

- RCTs that investigated the effects and safety of camrelizumab monotherapy and combined therapy.

\section{Exclusion criteria:}

- Systematic reviews without meta-analyses.

- Animal or in vitro models.

- No peer-reviewed article.

- Conference abstracts.

- Unable to extract valid data.

\section{Data Screening and Data Extraction}

Duplicates exclusion was implemented by two independent reviewers. If there was no consensus, the conflict was solved by a third reviewer. Two independent investigators extracted 
the following information from each article: (I) publication time; (II) corresponding author and first author; (III) PMID/ DOI; (IV) population and main condition of patients in RCT; (V) number of included studies and the total number of people included in the meta-analysis; (VI) study design of included primary studies (e.g., case-control, prospective, RCT); (VII) number of cases and controls for each study; (VIII) mean age of participant population; (IX) primary effectiveness index; (X) primary safety index.

\section{Risk of Bias and Quality Assessment}

The modified Jadad Scoring Scale (16) was used to evaluate the quality of eligible literature methodology with a score system of 1 to 7. Random sequence generation, blind method, randomized allocation concealment, and patient withdrawal were evaluated. Jadad scores of 4 to 7 were high-quality literature, and 1 to 3 were low-quality literature. The Cochrane risk of bias assessment tool was used to assess the methodological quality of individual studies based on the following aspects: random sequence generation, allocation concealment, blinding of participants and personnel, blinding of outcome and assessment, incomplete outcome data, selective reporting, and other bias. Each item was answered with high, low, or unclear risk of bias, and disagreements were resolved through an open discussion or a third reviewer. The general chart of bias risk was made by Revman software.

\section{Statistical Analysis}

Stata 12.0 software was used for meta-analysis. The binary variables were expressed by odds ratio (RR) and 95\% confidence interval (CI); the continuous variables were represented by standardized mean difference (SMD) and 95\% CI. If there was no statistical heterogeneity among the studies $(\mathrm{P}>0.1, \mathrm{I} 2<50 \%)$, the fixedeffects model was used for analysis; otherwise, the random effect model was used for analysis. Revman 5 software was used to map the risk of publication bias, and Egg's test was used to analyze publication bias. $\mathrm{P}<0.05$ was statistically significant.

\section{RESULTS}

\section{Systematic Literature Search}

The flowchart of PRISMA was shown in Figure 1. A total of 367 potentially relevant articles were included in the combined electronic and paper reference search. After preliminary screening, 333 publications were excluded according to the title and abstract. After detailed reading and evaluation of the full text, another 30 articles were excluded because they had no valid data to extract, or they were animal or basic research, or they were reviews without meta-analysis, or they were conference abstracts. Overall, four observational studies, involving 1233 patients with camrelizumab monotherapy and combination therapy, were included.

\section{Study Characteristics and Quality Assessment}

The details of the drug interventions, baseline characteristics of the populations, average age, study period, efficacy Index, and safety index of four eligible trials were shown in Table 1. The improved Jadad Scale (16) was used to evaluate its quality. The Jadad scores were between five and six points and were all highquality documents (Table 1).

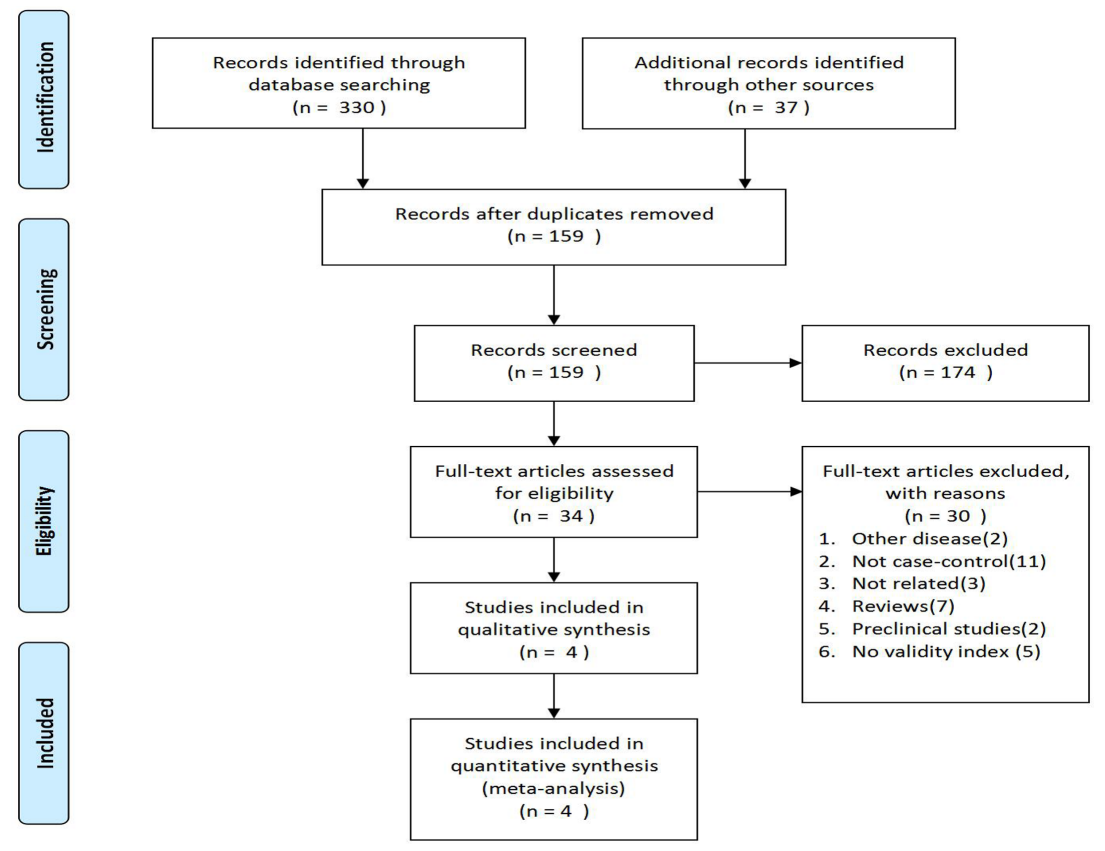

FIGURE 1 | Flowchart of studies evaluating qualified research through selection process. 
TABLE 1 | Characteristics of included studies and Jadad scores.

\begin{tabular}{|c|c|c|c|c|c|c|}
\hline Study & Jadad & Intervention & $\mathbf{n}$ & Year & Study period & Efficacy index and Safety index \\
\hline Wenfeng Fang (8) & 6 & $\begin{array}{l}\text { Camrelizumab + gemcitabine + cisplatin vs } \\
\text { Camrelizumab }\end{array}$ & 23/93 & $18-70$ & 12 months & ORR, PFS, adverse event rate (grade 1-5) \\
\hline Jing Nie (17) & 5 & Camrelizumab+ Decitabine vs Camrelizumab & $42 / 19$ & $18-75$ & 24 months & ORR, PFS, CR, adverse event rate (grade 1-5) \\
\hline Jing Huang (18) & 6 & Camrelizumab vs docetaxel or irinotecan & $228 / 220$ & $18-75$ & 24 months & ORR, PFS, CR, adverse event rate (grade 1-5) \\
\hline Caicun Zhou (19) & 6 & $\begin{array}{l}\text { Camrelizumab + Carboplatin + Pemetrexed vs } \\
\text { Carboplatin + Pemetrexed }\end{array}$ & $205 / 207$ & $18-70$ & 22 months & PFS, adverse event rate (grade 1-5) \\
\hline
\end{tabular}

\section{Efficacy Analysis}

The results of the meta-analysis of effectiveness indicators were as follows: the ORR, PFS, and CR value of combined treatment with camrelizumab were better than single camrelizumab treatment, camrelizumab alone was better than chemotherapy $[\mathrm{RR}=0.44,95 \% \mathrm{CI}(0.30,0.66), \mathrm{P}<0.001 ; \mathrm{RR}=1.63,95 \% \mathrm{CI}(1.25$, 2.13), $\mathrm{P}<0.001$; $\mathrm{RR}=0.73$, 95\%CI $(0.52,1.02), \mathrm{P}<0.001](8,17$ 19). The abovementioned results suggested that the combination of camrelizumab was better than monotherapy, and monotherapy was better than chemotherapy (Figure 2).

\section{Safety Analysis}

The results of meta-analysis of safety indicators were as follows: when the adverse event was grades 1 to 2 , there was no difference in incidence between camrelizumab monotherapy and combination in NPC [ OR $=0.97,95 \% \mathrm{CI}(0.51,1.85), \mathrm{P}>0.01]$, while the incidence rate of camrelizumab monotherapy was higher than chemotherapy in $\mathrm{EC}[\mathrm{OR}=1.49,95 \% \mathrm{CI}(1.10,2.01), \mathrm{P}<0.01](17,18)$. When adverse event was grade $>2$, the incidence of camrelizumab combination group was higher than chemotherapy $[\mathrm{OR}=1.25$, $95 \%$ CI $(0.85,1.84), \mathrm{P}<0.01]$, meanwhile the incidence of camrelizumab combination group and chemotherapy were higher than camrelizumab monotherapy [OR $=0.17,95 \% \mathrm{CI}(0.08,0.38)$,
$\mathrm{P}<0.01 ; \mathrm{OR}=0.49,95 \% \mathrm{CI}(0.32,0.73), \mathrm{P}<0.01 ; \mathrm{OR}=0.42,95 \% \mathrm{CI}$ $(0.12,1.55), \mathrm{P}<0.01](8,17-19)$ (Figure 3).

Using camrelizumab to treat cancers could cause some common adverse events (any grade), including reactive capillary hyperplasia, elevated aspartate aminotransferase, elevated alanine aminotransferase, anemia, elevated serum bilirubin, leukopenia, and hypothyroidism. From direct comparison, we found that the incidence of reactive capillary hyperplasia, elevated aspartate aminotransferase, elevated alanine aminotransferase, hypothyroidism, elevated serum bilirubin in single drug group was higher than the combination group. The incidence of anemia and leukopenia in the singledrug group were lower than the combination group. The abovementioned results showed that in terms of the incidence of common adverse events (any grade), the safety of combination therapy was better than that of monotherapy $(8,17)$ (Table 2). We also found that the incidence of reactive capillary hyperplasia, elevated aspartate aminotransferase, elevated alanine aminotransferase, anemia, elevated serum bilirubin, leukopenia, hypothyroidism in camrelizumab combination were higher than chemotherapy, suggesting that the safety of chemotherapy was better than camrelizumab combination (19) (Table 3).

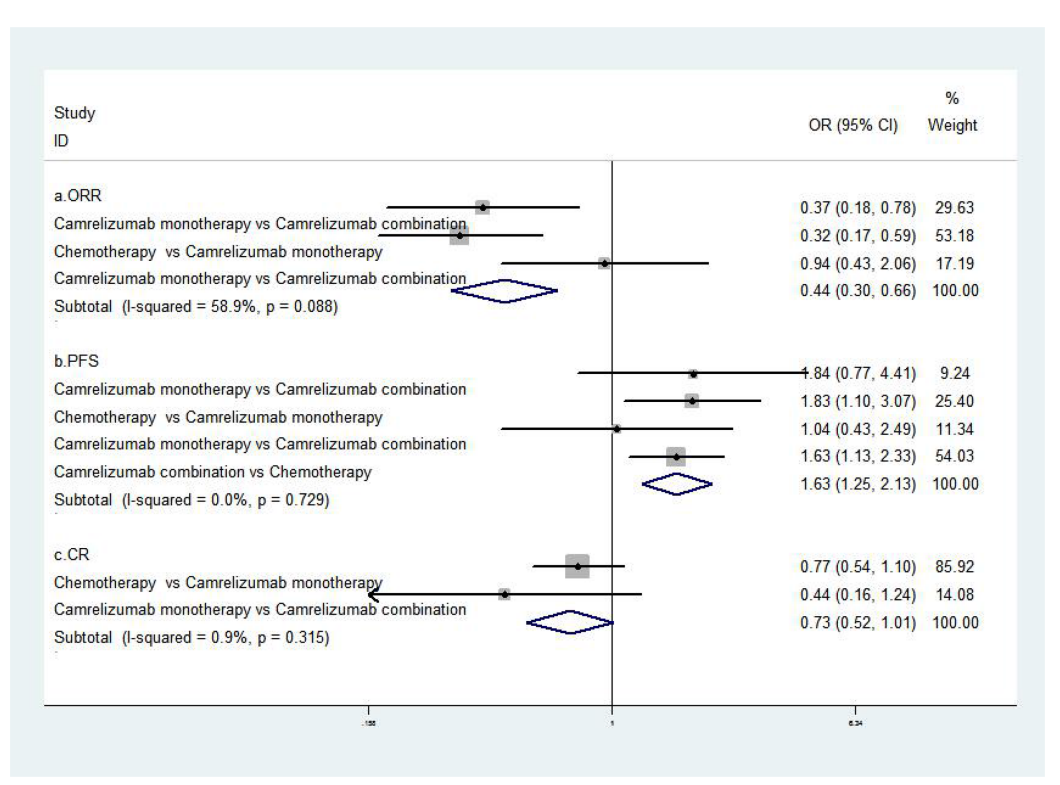

FIGURE 2 | Meta-analysis of efficacy of single and combined treatment of camrelizumab. 


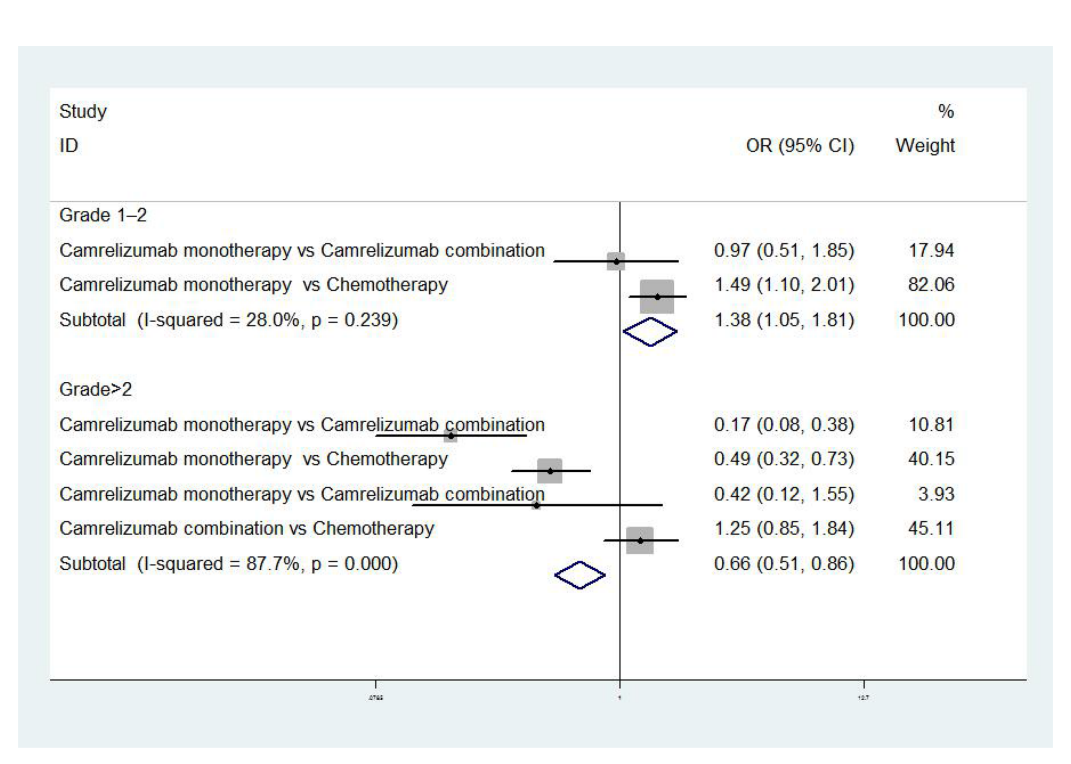

FIGURE 3 | Meta-analysis of safety of single and combined treatment of camrelizumab.

TABLE 2 | Comparison of the incidence of common adverse events (any grade) between camrelizumab alone and combination therapy.

\begin{tabular}{lccc}
\hline $\begin{array}{l}\text { Common adverse } \\
\text { event }\end{array}$ & $\begin{array}{c}\text { Camrelizumab combination } \\
\text { (incidence rate) }\end{array}$ & & $\begin{array}{c}\text { Camrelizumab alone } \\
\text { (incidence rate) }\end{array}$ \\
\cline { 2 - 2 } $\begin{array}{lcc}\text { Reactive capillary } \\
\text { hyperplasia }\end{array}$ & $22 \%$ & & Any grade \\
\hline $\begin{array}{l}\text { Elevated aspartate } \\
\text { aminotransferase }\end{array}$ & $17 \%$ & $88 \%$ \\
$\begin{array}{l}\text { Elevated alanine } \\
\text { aminotransferase }\end{array}$ & $14 \%$ & $48 \%$ \\
$\begin{array}{l}\text { Anemia } \\
\begin{array}{l}\text { Elevated serum } \\
\text { bilirubin }\end{array}\end{array}$ & $100 \%$ & & \\
Leukopenia & $0 \%$ & & \\
Hypothyroidism & $87 \%$ & & $13 \%$ \\
\hline
\end{tabular}

TABLE 3 | Comparison of the incidence of common adverse events (any grade) between camrelizumab combination and chemotherapy.

\begin{tabular}{|c|c|c|}
\hline \multirow[t]{2}{*}{$\begin{array}{l}\text { Common adverse } \\
\text { event }\end{array}$} & $\begin{array}{l}\text { Camrelizumab combination } \\
\text { (incidence rate) }\end{array}$ & $\begin{array}{l}\text { Chemotherapy } \\
\text { (incidence rate) }\end{array}$ \\
\hline & Any grade & Any grade \\
\hline $\begin{array}{l}\text { Reactive capillary } \\
\text { hyperplasia }\end{array}$ & $78 \%$ & $<1 \%$ \\
\hline $\begin{array}{l}\text { Elevated aspartate } \\
\text { aminotransferase }\end{array}$ & $47 \%$ & $33 \%$ \\
\hline $\begin{array}{l}\text { Elevated alanine } \\
\text { aminotransferase }\end{array}$ & $48 \%$ & $40 \%$ \\
\hline Anemia & $34 \%$ & $27 \%$ \\
\hline Elevated serum bilirubin & $11 \%$ & $5 \%$ \\
\hline Leukopenia & $91 \%$ & $78 \%$ \\
\hline Hypothyroidism & $11 \%$ & $0 \%$ \\
\hline
\end{tabular}

\section{Heterogeneity and Risk of Bias}

The fixed-effect model was used to analyze the efficacy and safety indicators, and the heterogeneity generally met the requirements. However, the heterogeneity was high at an incidence rate of grade $>2$. After the first study was excluded, the heterogeneity could meet the requirements. ORR, PFS, and CR were used as indicators $(8,17-19)$ for publication bias analysis. Egger's test was used for publication bias analysis. The standard error of logarithm of OR value of each independent study was taken as abscissa and logarithm of OR value of each independent study as ordinate. A funnel plot was drawn, as shown in Figure 4. Egger's test $(\mathrm{P}=0.905>0.05)$ indicated that there was less possibility of publication bias. The general chart of bias risk was shown in Figure 5.

\section{DISCUSSION}

Recently, there is now growing worldwide enthusiasm in cancer immunotherapy (20). ICI are the main drugs of immunotherapy and have recently emerged as a promising treatment for many cancers, which identified to target the cytotoxic T lymphocyteassociated antigen-4 (CTLA-4) or PD-1/PD-L1 pathways (21). One of the ICI named camrelizumab (AiRuiKa ${ }^{\mathrm{TM}}$ ), is a PD-1 inhibitor being developed by Jiangsu Hengrui Medicine Co. Ltd (6). In May 2019, camrelizumab for injection was conditionally approved by the State Drug Administration of China for marketing. The indication was the treatment of patients with relapsed or refractory classical HDL who had received at least second-line systemic chemotherapy (12). In 2020, it was approved for the treatment of patients with advanced HCC who had previously received sorafenib treatment and/or oxaliplatin containing systemic chemotherapy, combination of pemetrexed, 


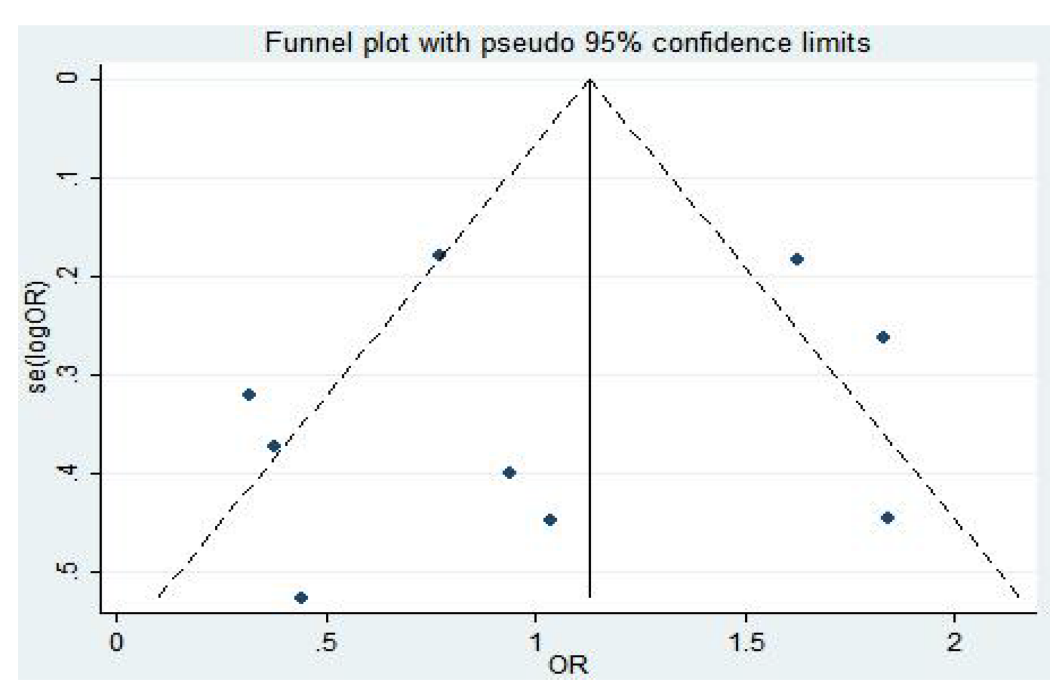

FIGURE 4 | Funnel diagram based on ORR, PFS, CR.

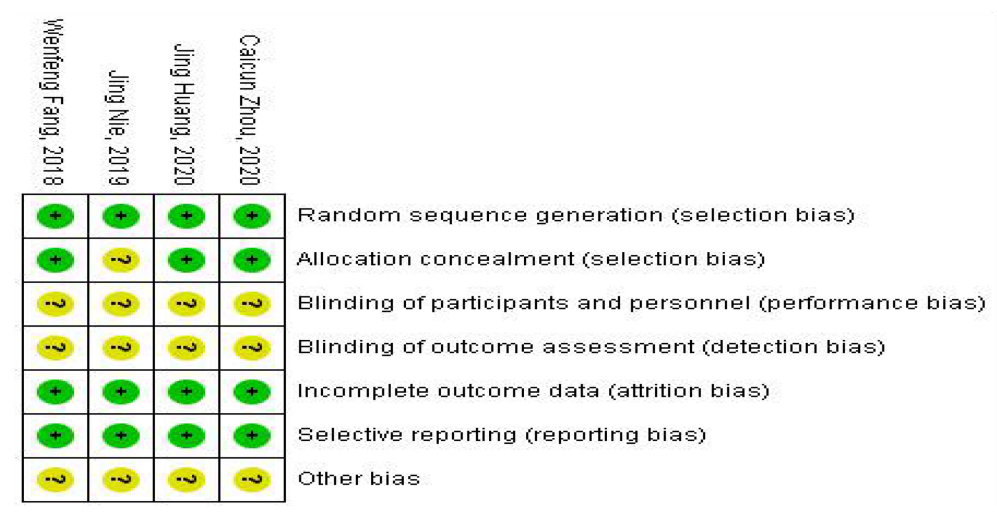

FIGURE 5 | Summary chart of bias risk.

and carboplatin for the first-line treatment of unresectable locally advanced or metastatic NSCLC with negative epidermal growth factor receptor (EGFR) gene mutation and negative anaplastic lymphoma kinase (ALK), and for the treatment of patients with locally advanced or metastatic ES who had previously received first-line chemotherapy $(22,23)$. In 2020, camrelizumab was recommended by the Chinese Society of Clinical Oncology (CSCO) clinical guidelines for the treatment of NSCLC, HCC, $\mathrm{EC}$, and HDL and became the only immunotherapy drug to achieve this achievement in China (24). In 2021, camrelizumab was approved by FDA as orphan drug (14). Orphan drug, also known as rare disease drug, refers to the drugs used to prevent, treat, and diagnose rare diseases, the candidate drugs who obtained the orphan drug qualification had the opportunity to obtain a series of supporting policies (25). After obtaining the orphan drug qualification, the clinical trials and market registration can be accelerated, at the same time, the clinical trial fee can be partly offset by tax, the new drug registration fee can be reduced, and the product will enjoy 7 years of market monopoly after being approved (26). Moreover, PubMed, Web of Science, and other literature search websites did not find a meta-analysis of the comparison between single-drug and combination drug of camrelizumab, so this study had certain importance and innovation. Therefore, it can be seen that camrelizumab has a good development prospect and it is necessary to carry out RCTs of single and combined treatment of camrelizumab; however, the RCTs for HCC were in progress, and no clinical results were available, so this meta-analysis included four indications, which were NSCLC, NPC, ES, and HDL.

In the aspect of effectiveness indicators, we found that the ORR, PFS, CR value of combined treatment with camrelizumab were better than that of single-drug treatment, and the ORR, PFS, CR value of single-drug treatment with camrelizumab were better than that of chemotherapy. These results indicated that the 
combination therapy of camrelizumab was more effective than single drug or chemotherapy $(8,17-19)$.

In the aspect of safety index, the severity of adverse event was divided into five grades. The most common adverse reactions were reactive capillary hyperplasia (77.4\%), elevated aspartate aminotransferase (19.0\%), elevated alanine aminotransferase (17.5\%), hypothyroidism (16.7\%), fatigue (15.3\%), anemia (14.1\%), elevated serum bilirubin $(11.5 \%)$, proteinuria $(10.8 \%)$, fever $(10.4 \%)$, and leukopenia $(10 \%)(27,28)$. Through metaanalysis, we found that when the grade of adverse events was $>2$, the incidence of single use was lower than combination therapy and chemotherapy. However, according to the incidence of common adverse reactions (Any grade), the safety of combined use of camrelizumab was better than that of single use, and the safety of chemotherapy was better than the combined use of camrelizumab plus chemotherapy (8, 17-19).

However, this meta-analysis still has some limitations. First, the sample size of RCTs of camrelizumab monotherapy and combination therapy is small because of the lack of existing research. Second, some included studies are lack of double-blind implementation, so there may be selection bias. Finally, although we considered the studies with lower levels of validity, the number was small, and there was the possibility for lack of robustness. Therefore, this conclusion needs to be further confirmed by more high-quality, multi-center, and large-sample researches.

\section{CONCLUSION}

Overall, in terms of effectiveness, the combination of camrelizumab is better than monotherapy, and monotherapy is better than chemotherapy. In terms of safety, when the grade of adverse events $>2$, single use is better than combination therapy and chemotherapy. However, in any grade, the safety of combined use of camrelizumab is better than that of single use,

\section{REFERENCES}

1. Kroemer G, Zitvogel L. Immune Checkpoint Inhibitors. J Exp Med (2021) 218 (3):e20201979. doi: 10.1084/jem.20201979

2. Kim SI, Cassella CR, Byrne KT. Tumor Burden and Immunotherapy: Impact on Immune Infiltration and Therapeutic Outcomes. Front Immunol (2021) 11:629722. doi: 10.3389/fimmu.2020.629722

3. Yang Y, Yang J, Shen L, Chen J, Xia L, Ni B, et al. A Multi-Omics-Based Serial Deep Learning Approach to Predict Clinical Outcomes of Single-Agent AntiPD-1/PD-L1 Immunotherapy in Advanced Stage Non-Small-Cell Lung Cancer. Am J Transl Res (2021) 13(2):743-56.

4. Voutsadakis IA. PD-1 Inhibitors Monotherapy in Hepatocellular Carcinoma: Meta-Analysis and Systematic Review. Hepatobiliary Pancreat Dis Int (2019) 9(3):261-72. doi: 10.1016/j.hbpd.2019.09.007

5. Heymach J, Krilov L, Alberg A, Baxter N, Chang SM, Corcoran RB, et al. Clinical Cancer Advances 2018: Annual Report on Progress Against Cancer From the American Society of Clinical Oncology. J Clin Oncol (2018) 36:1020-44. doi: 10.1200/JCO.2017.77.0446

6. Markham A, Keam SJ. Camrelizumab: First Global Approval. Drugs (2019) 79 (12):1355-61. doi: 10.1007/s40265-019-01167-0

7. Mo H, Huang J, Xu J, Chen X, Wu D, Qu D, et al. Safety, Anti-Tumour Activity, and Pharmacokinetics of Fixed-Dose SHR-1210, An Anti-PD-1 and the safety of chemotherapy is better than the combined use of camrelizumab plus chemotherapy. Therefore, when the patient with serious clinical course and the physical status is tolerable, the combination of camrelizumab is more recommended. When the patient has poor healthy condition, monotherapy is recommended; however, large sample studies are still needed to further prove this inference.

\section{DATA AVAILABILITY STATEMENT}

The datasets presented in this study can be found in online repositories. The names of the repository/repositories and accession number(s) can be found below: Embase, MEDLINE/ Ovid, Epistemonikos and Cochran, accession numbers T5M3L2NLFV,13882707759,20190599120027.

\section{AUTHOR CONTRIBUTIONS}

JW and YL carried out the conception and design of the article and wrote the paper. JW and JL carried out the implementation and feasibility analysis of the research. JW and SS collected the data. SS carried out the data sorting. JW carried out the statistical processing. JL carried out the analysis and interpretation of the results, and the revision of the paper. YL was responsible for the quality control and review of the article, and analyzed the overall article. All authors contributed to the article and approved the submitted version.

\section{FUNDING}

This study was supported by the National Natural Science Foundation of China (81803019) and Sichuan Provincial Department of Education (SCYG2019-04, YF19-Y12).
Antibody in Advanced Solid Tumours: A Dose-Escalation, Phase 1 Study. Br J Cancer (2018) 119:538-45. doi: 10.1038/s41416-018-0100-3

8. Fang W, Yang Y, Ma Y, Hong S, Lin L, He X, et al. Camrelizumab (SHR-1210) Alone or in Combination With Gemcitabine Plus Cisplatin for Nasopharyngeal Carcinoma: Results From Two Single-Arm, Phase 1 Trials. Lancet Oncol (2018) 19(10):1338-50. doi: 10.1016/S1470-2045(18)30495-9

9. Xu J, Zhang Y, Jia R, Yue C, Chang L, Liu R, et al. Anti-PD-1 Antibody Shr-1210 Combined With Apatinib for Advanced Hepatocellular Carcinoma, Gastric, or Esophagogastric Junction Cancer: An Open-Label, Dose Escalation and Expansion Study. Clin Cancer Res (2019) 25:515-23. doi: 10.1158/1078-0432.CCR-18-2484

10. Song H, Liu X, Jiang L, Li F, Zhang R, Wang P. Current Status and Prospects of Camrelizumab, A Humanized Antibody Against Programmed Cell Death Receptor 1. Recent Pat Anticancer Drug Discovery (2021) 16:133. doi: 10.2174/ 1574892816666210208231744

11. Rakké YS, Sprengers D, Kwekkeboom J, IJzermans JNM. CamrelizumabTargeting a Novel PD-1 Epitope to Treat Hepatocellular Carcinoma. Ann Transl Med (2020) 8(23):1614. doi: 10.21037/atm-2020-115

12. Cohen AC, Roane BM, Leath CA3rd. Novel Therapeutics for Recurrent Cervical Cancer: Moving Towards Personalized Therapy. Drugs (2020) 80 (3):217-27. doi: 10.1007/s40265-019-01249-z

13. Mei K, Qin S, Chen Z, Liu L, Wang L, Zou J, et al. Camrelizumab in Combination With Apatinib in Second-Line or Above Therapy for Advanced 
Primary Liver Cancer: Cohort A Report in a Multicenter Phase Ib/II Trial. J Immunother Cancer (2021) 9(3):e002191. doi: 10.1136/jitc-2020-002191

14. Uson Junior PLS, Liu AJ, Sonbol MB, Borad MJ, Bekaii-Saab TS, et al. Immunotherapy and Chimeric Antigen Receptor T-Cell Therapy in Hepatocellular Carcinoma. Chin Clin Oncol (2021) 10(1):11. doi: 10.21037/cco-20-231

15. Hu Y, Qin T, Li S, Zhang T, Xue J. Efficacy and Safety of SBRT Combined With Camrelizumab and Apatinib in HCC Patients With Pvtt: Study Protocol of a Randomized Controlled Trial. Front Oncol (2020) 10:1589. doi: 10.3389/ fonc.2020.01589

16. Clark HD, Wells GA, Huët C, McAlister FA, Salmi LR, Fergusson D, et al. Assessing the Quality of Randomized Trials: Reliability of the Jadad Scale. Control Clin Trials (1999) 20(5):448-52. doi: 10.1016/S0197-2456(99)00026-4

17. Nie J, Wang C, Liu Y, Yang Q, Mei Q, Dang L, et al. Addition of Low-Dose Decitabine to Anti-PD-1 Antibody Camrelizumab in Relapsed/Refractory Classical Hodgkin Lymphoma. J Clin Oncol (2019) 37(17):1479-89. doi: 10.1200/JCO.18.02151

18. Huang J, Xu J, Chen Y, Zhuang W, Zhang Y, Chen Z, et al. Camrelizumab Versus Investigator's Choice of Chemotherapy as Second-Line Therapy for Advanced or Metastatic Oesophageal Squamous Cell Carcinoma (ESCORT): A Multicentre, Randomised, Open-Label, Phase 3 Study. Lancet Oncol (2020) 21(6):832-42. doi: 10.1016/S1470-2045(20)30110-8

19. Zhou C, Chen G, Huang Y, Zhou J, Lin L, Feng J, et al. Camrelizumab Plus Carboplatin and Pemetrexed Versus Chemotherapy Alone in ChemotherapyNaive Patients With Advanced Non-Squamous Non-Small-Cell Lung Cancer (CameL): A Randomised, Open-Label, Multicentre, Phase 3 Trial. Lancet Respir Med (2021) 9(3):305-14. doi: 10.1016/S2213-2600(20)30365-9

20. Steven A, Fisher SA, Robinson BW. Immunotherapy for Lung Cancer. Respirology (2016) 21(5):821-33. doi: 10.1111/resp.12789

21. Ravindran N, Thuluvath PJ. A Large Randomized Clinical Trial Is Necessary to Establish the Role of Camrelizumab in Hepatocellular Carcinoma. Ann Transl Med (2020) 8(19):1253. doi: 10.21037/atm-2020-71
22. Fan Y, Zhao J, Wang Q, Huang D, Li X, Chen J, et al. Camrelizumab Plus Apatinib in Extensive-Stage SCLC (Passion): A Multicenter, Two-Stage, Phase 2 Trial. J Thorac Oncol (2021) 16(2):299-309. doi: 10.1016/j.jtho.2020.10.002

23. Chen Z, Lu X, Koral K. The Clinical Application of Camrelizumab on Advanced Hepatocellular Carcinoma. Expert Rev Gastroenterol Hepatol (2020) 14(11):1017-24. doi: 10.1080/17474124.2020.1807939

24. Masterson L, Howard J, Gonzalez-Cruz J, Jackson C, Barnett C, Overton L, et al. Immune Checkpoint Inhibitors in Advanced Nasopharyngeal Carcinoma: Beyond an Era of Chemoradiation? Int J Cancer (2020) 146 (8):2305-14. doi: 10.1002/ijc.32869

25. Blin O, Lefebvre MN, Rascol O, Micallef J. Orphan Drug Clinical Development. Therapie (2020) 75(2):141-7. doi: 10.1016/j.therap.2020.02.004

26. Weerasooriya SU. The Impact of Orphan Drug Policies in Treating Rare Diseases. Health Info Libr J (2019) 36(2):179-84. doi: 10.1111/hir.12256

27. Teng Y, Guo R, Sun J, Jiang Y, Liu L. Reactive Capillary Hemangiomas Induced by Camrelizumab (SHR-1210), an Anti-PD-1 Agent. Acta Oncol (2019) 58(3):388-9. doi: 10.1080/0284186X.2019.1567935

28. Song P, Zhang D, Cui X, Zhang L. Meta-Analysis of Immune-Related Adverse Events of Immune Checkpoint Inhibitor Therapy in Cancer Patients. Thorac Cancer (2020) 11(9):2406-30. doi: 10.1111/1759-7714.13541

Conflict of Interest: The authors declare that the research was conducted in the absence of any commercial or financial relationships that could be construed as a potential conflict of interest.

Copyright (c) 2021 Wang, Su, Li and Li. This is an open-access article distributed under the terms of the Creative Commons Attribution License (CC BY). The use, distribution or reproduction in other forums is permitted, provided the original author(s) and the copyright owner(s) are credited and that the original publication in this journal is cited, in accordance with accepted academic practice. No use, distribution or reproduction is permitted which does not comply with these terms. 